\title{
Swift contribution to our understanding of Ultraluminous X-ray Sources
}

\author{
Luca Zampieri ${ }^{1}$ \\ INAF-Astronomical Observatory of Padova \\ Vicolo dell'Osservatorio 5, 35122, Padova, Italy \\ E-mail: Iuca.zampieri@oapd.inaf.it
}

In the coming years a significant improvement in our understanding of Ultraluminous X-ray Sources (ULXs) is expected to come from the study of their long term X-ray variability and from the discovery and follow up of the still largely unknown population of transient sources. This requires a flexible and fast scheduling strategy to monitor ULXs and observe them in 'ToO' mode. Swift has shown to be the best suited X-ray facility for this purpose, capable to do effectively both things for bright ULXs. After a short summary on the properties of ULXs and the recent advances made in this field, I will focus on the contribution given by Swift, reviewing the main results obtained by this extraordinarily successful mission.

Swift: 10 Years of Discovery

2-5 December 2014

La Sapienza University, Rome, Italy

${ }^{1}$ Speaker 


\section{The 'manifold population' of Ultraluminous X-ray Sources}

Although the first very luminous X-ray sources in the field of nearby galaxies were already noticed in EINSTEIN data, it was only with ROSAT, XMM-Newton and Chandra that an entire population of intrinsically powerful, but faint, point-like off-nuclear X-ray sources, nowadays called Ultraluminous X-ray sources (ULXs), has been largely uncovered. Their luminosity exceeds (although not necessarily all the time) the Eddington limit for spherical accretion onto a $\sim 10$ solar mass black hole $\left(\mathrm{L}>10^{39} \mathrm{erg} / \mathrm{s}\right)$. Hundreds of sources are reported in various surveys/catalogues (ROSAT, e.g. [1,2]; Chandra [3]; XMM-Newton [4]). While a significant fraction of ULXs $(\sim 20 \%)$ are background AGNs, the majority of the population is believed to be accreting binaries, with a modest contamination ( $5 \%$ ) from supernovae interacting with the circumstellar medium. This conclusion comes from several observational evidences, including the X-ray emission properties, the frequent association with young stellar environments, the detection of stellar optical counterparts, the relation with the properties (such as star formation rate and X-ray luminosity function) of their host galaxies (see e.g. [5,6]).

In a few cases a periodic modulation has been detected in the X-ray or optical light curve (although in a couple of cases the statistical significance may not be very large) and interpreted as the orbital period of the system. Observed periods range from $\sim 6 \mathrm{hr}$ to $\sim 60$ days [7,8,9]. Most important, the first measurements of the mass function of two ULXs have been recently published (M 101 ULX-1 [10]; ULX P13 in NGC 7793 [11]). They demonstrate both the binary nature of these sources and that at least some of them host stellar black holes. In fact, we now know of a ULX that shows $\sim 1 \mathrm{~s}$ X-ray pulsations modulated with a very short orbital period of 2.5 days and thus contains a neutron star (M82 X-2 [12]).

\subsection{ULXs: questions, interpretations and implications}

Despite the significant advances made in this field in the last years, many important questions still challenge our interpretation of ULXs. What are the masses and origin of the black holes (BHs) hosted in ULXs? How can a neutron star (NS) power them? What may ULXs tell us about intermediate mass BHs? How extreme is their accretion environment?

Our present understanding suggests that ULXs are the key to exploring the unknown distribution of $\mathrm{BH}$ masses above 10 solar masses in the local Universe. Some of them may contain the long sought intermediate mass BHs ( $>100$ solar masses [13]), that may form through repeated mergers of stellar mass BHs [14] or from the dynamical collapse of a supermassive star in dense stellar clusters [15]. They may also be the relics of the collapse of Population III stars and the seeds of the first supermassive BHs [16]. But the majority of them probably contain BHs of stellar origin, either stellar-mass $(<20$ solar masses [17]) or more massive $(20-80$ solar masses $[5,18,19])$. In the latter case, the accretion rate needed to sustain the observed luminosity most likely requires accretion to proceed at super-Eddington rates. The physical conditions in ULXs could then be similar to those occurring in the first generation of Quasars at very high redshift. 


\section{ULXs in the X-rays}

\subsection{X-ray spectra}

The significant amount of high quality X-ray spectra of ULXs collected by XMMNewton in the last 10 years turned out to be essential to clarify subtle but important differences with respect to the spectra of Galactic BH X-ray binaries. Many ULXs show either curved X-ray spectra or a turnover at $\sim 3-5 \mathrm{keV}$, sometimes with a soft excess below $1 \mathrm{keV}$ (ultraluminous state [20]). The validity of these two-component thermal models has been recently confirmed by broadband XMM+NuSTAR observations (e.g. [21]). Spectra in the ultraluminous state are interpreted in terms of an optically thick corona covering or replacing the disc, or a modified accretion disc (like a slim disc) that is launching a wind (e.g. [22]).

\subsection{X-ray spectral evolution: Super-Eddington accretion?}

Spectral variability is frequently detected and appears to be rather complex (e.g. $[23,24,25])$. The observed spectral evolution may be satisfactorily reproduced by changes in an optically thick thermal component (corona/wind) and an accretion disc, energetically coupled to it [26,27]. Both components appear to be in unusual physical conditions, likely associated to the accretion rate being significantly above the Eddington limit. The corona/wind along the line of sight causes increased variability in the high energy part of the spectrum on a timescale of hundreds of seconds [28]. However, the existence of such strong winds is challenged by the lack of detection of Xray absorption features [29], although it has been suggested that broadened and blueshifted absorption lines may be consistent with the soft residuals seen in the X-ray spectra of two sources [30].

\subsection{Short-term X-ray variability}

The timing behaviour of ULXs is heterogeneous. Variability on short time scales (below $10 \mathrm{~s}$ ) is often absent [31]. However, some ULXs have features in the power density spectrum (PDS) in the form of low-frequency broad-band noise and/or quasi periodic oscillations (QPOs). The frequencies of the QPOs are in the range from $\sim 1 \mathrm{mHz}$ to a few Hz: 54-166 mHz, $3.32 \mathrm{~Hz}$ and $5.07 \mathrm{~Hz}$ in M82 X-1 ([32,33,34]), $20 \mathrm{mHz}$ in NGC 5408 X-1 [35], $642 \mathrm{mHz}$ in IC 342 X-1 [36], 3-4 mHz in M82 X42.3+59 [37].

The detection of QPOs has prompted attempts to compare them to similar features in Galactic $\mathrm{BH}$ binaries and to estimate the $\mathrm{BH}$ mass by scaling their characteristic frequencies or by extrapolating correlations known to exist for Galactic binaries (e.g. $[33,35,38])$. For M82 X-1 the frequencies of the two QPOs above $1 \mathrm{~Hz}$ appear to be in a ratio 3:2. An inverse-mass scaling of their frequencies with those of the high frequency $(100-450 \mathrm{~Hz})$ QPOs with a similar 3:2 ratio in Galactic BH binaries yields a mass of $\sim 400$ solar masses [34]. Other methods to estimate masses from timing include the nondetection of variability power [39] or the "variability plane" of both Galactic BH binaries and AGNs [40]. 


\section{Swift contribution}

Thanks to its flexible monitoring strategy, Swift has shown to be the best suited X-ray facility to search for long-term (months-to-years) periodicities and variability in ULXs. Here I will shortly summarize some of the most significant results obtained with Swift.

\subsection{Search for X-ray periodicities}

A dedicated monitoring campaign performed with Swift/XRT led to the discovery of a periodicity of 155.5 days in the light curve of NGC 5408 X-1 [41]. This value was later revisited in 243 days using 1240 days of monitoring of the source [42]. However, the result could not be confirmed by Grise' et al. [43] using an even longer baseline (1532 days). The significance of the detection decreases with time and the periodicity disappears after a few cycles. An equally interesting interpretation of this non-persistent modulation in terms of a super-orbital period caused by the precession of the innerdisc/jet in the system has been proposed by Foster et al. [44].

\subsection{Characterization of X-ray flux variability}

Monitoring with Swift/XRT is particularly successfull in identifying structured longterm variability in ULXs, with typical flux variations up to 5-10. Crucial to this end is the unprecedented temporal coverage and cadence made possible by Swift.

Extended periods of pronounced flaring activity were observed in Holmberg II X-1, that do not appear to be associated to significant spectral variability [45]. On the other hand, long low flux 'states' are observed in Holmberg IX X-1 [46], followed by active flaring phases. High-low flux 'states' are also seen in NGC 55 ULX-1, accompanied by spectral changes similar to those observed in brighter ULXs [47]. This low-luminosity ULX is rather peculiar because in two XMM-Newton observations it showed energy-dependent dips, not commonly observed in these sources [48]. Short drops in the count rate, but no clear evidence of dips on timescales longer than 300s were detected in Swift data, although the uncertainties due to the low counting statistics are large.

Another ULX well monitored by Swift is NGC 1313 X-2. A very extended observing campaign of this source, started in June 2013 and still ongoing, has led to the clear identification of flux-dependent activity (high-flaring/low-quiescient phases). The quality of the data attained is such that we may be able to constrain also the possible timescales of these flux transitions (Grise' et al., in preparation).

\subsection{Follow-up of transients ULXs}

Another area in which the Swift contribution has been relevant is the monitoring of transient ULXs (e.g. the ULX in M 83 [49]). Particularly successfull was the follow-up of the second brigthest ULX in M31 (M31 ULX-2 hereafter) discovered in January 2012 thanks to the XMM-Newton/Chandra M31 nova monitoring programme [50]. Thanks to its fast and flexible scheduling, after discovery Swift started an immediate follow-up observing programme targeted to this source [51]. The source reached a peak luminosity of $\sim 10^{39} \mathrm{erg} / \mathrm{s}$ and for at least 40 days the luminosity remained fairly constant. Then, in the following 200 days, it faded below $10^{38} \mathrm{erg} / \mathrm{s}$. The Swift spectrum was well described by a multi-colour disc blackbody model, progressively softening during the decay (from $\mathrm{kT} \sim 0.9 \mathrm{keV}$ to $\sim 0.4 \mathrm{keV}$ ). Assuming that at maximum 
luminosity the source was in a disc dominated state and for moderate inclinations, the mass inferred from the normalisation of the multi-colour disc blackbody component is 12-14 solar masses.

M31 ULX-2 was jointly monitored in the radio and X-rays by several facilities. Simultaneous X-ray and radio measurements demonstrated a clear bright sub-Eddington state, with radio flaring (on timescales as short as minutes) analogous to that seen in the Galatic BH binary GRS $1915+105$, arguing that the source is highly compact and powered by accretion close to the Eddington limit onto a stellar mass black hole [52].

\subsection{HLX-1 in ESO 243-49: a recurrent hyper-luminous transient ULX}

The most luminous ULX and strongest intermediate mass $\mathrm{BH}$ condidate to date is the hyperluminous X-ray source HLX-1 in the galaxy ESO 243-49 [53,54,55]. It is a recurrent transient ULX that, at maximum, reaches a peak luminosity of $10^{42} \mathrm{erg} / \mathrm{s}$. Since its discovery, in 2009, it underwent 6 outburts, all followed by Swift. Luminositycorrelated spectral variability similar to that observed in Galactic BH binaries is seen in this source, with the X-ray spectrum at maximum well fitted by a multi-colour disc blackbody model [56]. The relatively low disk temperature in this disc-dominated state $(0.2-0.3 \mathrm{keV})$ suggests the presence of an intermediate mass BH with a mass of $\sim 20000$ solar masses (e.g. [57]). Detection of ballistic radio jets allows for an even higher mass between 9000 and 90000 solar masses [55].

The X-ray light curve of all the outbursts has been carefully sampled by Swift and reveals very interesting properties. The decay timescale is too short to be consistent with the thermal-viscous timescale of a standard disc around an intermediate mass $\mathrm{BH}$. Outbursts are instead believed to be induced by bursts of mass transfer occurring when the donor, in an eccentric orbit, grazes the tidal radius [58]. However, the size of the accretion disc inferred from optical-through-X-ray spectral fitting turns out to be too large for the observed decay time [59]. Furthermore, the interval between successive outbursts seems to increase with time and it may not be strictly periodic. These fact make the intepretation of the feeding mechanism of the intermediate mass $\mathrm{BH}$ still debated.

\section{Conclusions}

Thanks to its unprecedented coverage Swift has allowed us to: place constraints on long-term periodicities (orbital and super-orbital periods) in ULXs; start characterizing their long-term X-ray flux variability (flux transitions, flaring activity, timescales); follow-up transients ULXs, including the remarkable transients in M31 and the recurrent hyper-luminous transient and intermediate mass BH candidate HLX-1 in ESO 243-49.

In the coming years a significant improvement in our understanding of ULXs is expected to come from the study of their long term X-ray variability and from the discovery and follow up of the still largely unknown population of transient sources. This requires a flexible and fast scheduling strategy. Swift has shown to be the best suited X-ray facility for this purpose. 


\section{Acknowledgments}

We acknowledge financial support from the INAF research grant PRIN-2011-1 ("Challenging Ultraluminous X-ray sources: chasing their black holes and formation pathways") and financial contribution from the agreement ASI-INAF I/037/12/0.

\section{References}

[1] J.-F. Liu \& J. N. Bregman, Ultraluminous X-Ray Sources in Nearby Galaxies from ROSAT High Resolution Imager Observations I. Data Analysis, ApJS 157 (2005) 59

[2] Q. Z. Liu \& I.F. Mirabel, A catalogue of ultraluminous X-ray sources in external galaxies, A\&A 429 (2005) 1125

[3] D. A. Swartz et al., A Complete Sample of Ultraluminous X-ray Source Host Galaxies, ApJ 741 (2011) id. 49

[4] D. J. Walton et al., 2XMM ultraluminous X-ray source candidates in nearby galaxies, MNRAS 416 (2011) 1844

[5] L. Zampieri \& T. P. Roberts 2009, Low-metallicity natal environments and black hole masse in ultraluminous X-ray sources, MNRAS 400 (2009) 677

[6] H. Feng \& R. Soria, Ultraluminous X-ray sources in the Chandra and XMM-Newton era, New Astronomy Reviews, 55 (2011) 166

[7] P. Kaaret et al., The orbital period of the ultraluminous X-ray source in M 82, Sci 311 (2006) 491

[8] J.-F. Liu et al., Hubble Space Telescope Monitoring Reveals a 6.1 Day Period for an Ultraluminous $X$-Ray Source in NGC 1313, ApJL 690 (2009) L39

[9] P. Esposito et al., Discovery of a 6.4 h black hole binary in NGC 4490, MNRAS 436 (2013) 3380

[10] J.-F. Liu et al., Puzzling accretion onto a black hole in the ultraluminous X-ray source M 101 ULX1, Natur 503 (2013) 500

[11] C. Motch et al., A mass of less than 15 solar masses for the black hole in an ultraluminous X-ray source, Natur 514 (2014) 198

[12] M. Bachetti et al., An ultraluminous X-ray source powered by an accreting neutron star, Natur 514 (2014) 202

[13] E. J. M. Colbert \& R. F. Mushotzky, The Nature of Accreting Black Holes in Nearby Galaxy Nuclei, ApJ, 519 (1999) 89

[14] M. C. Miller \& D. P. Hamilton, Production of intermediate-mass black holes in globular clusters, MNRAS 330 (2002) 232

[15] S. F. Portegies-Zwart et al., Formation of massive black holes through runaway collisions in dense young star clusters, Natur 428 (2004) 724

[16] P. Madau \& M. J. Rees, Massive Black Holes as Population III Remnants, ApJ 551 (2001) L27

[17] A. R. King et al., Ultraluminous X-Ray Sources in External Galaxies, ApJ 552 (2001) L109

[18] M. Mapelli et al., Low metallicity and ultra-luminous X-ray sources in the Cartwheel galaxy, MNRAS 395 (2009) L71 
[19] K. Belczynski et al., On the Maximum Mass of Stellar Black Holes, ApJ 714 (2010) 1217

[20] J. C. Gladstone et al., The ultraluminous state, MNRAS 397 (2009) 1836

[21] M. Bachetti et al., The Ultraluminous X-Ray Sources NGC 1313 X-1 and X-2: A Broadband Study with NuSTAR and XMM-Newton 2013, ApJ 778 (2013) id. 163

[22] M. J. Middleton et al., X-ray spectral evolution in the ultraluminous X-ray source M33 X-8, MNRAS 417 (2011) 464

[23] H. Feng \& P. Kaaret, Spectral States and Evolution of Ultraluminous X-Ray Sources, ApJ 696 (2009) 1712

[24] J. J. E. Kajava \& J. Poutanen, Spectral variability of ultraluminous X-ray sources, MNRAS 398 (2009) 1450

[25] F. Pintore \& L. Zampieri, X-ray spectral states and metallicity in the ultraluminous X-ray sources NGC $1313 X-1$ and X-2, MNRAS 420 (2012) 1107

[26] A. D. Sutton et al., The ultraluminous state revisited: fractional variability and spectral shape as diagnostics of super-Eddington accretion, MNRAS 435 (2013) 1758

[27] F. Pintore et al., Ultraluminous X-ray sources: a deeper insight into their spectral evolution, MNRAS 439 (2014) 3461

[28] M. J. Middleton et al., Challenging times: a re-analysis of NGC 5408 X-1, MNRAS 411 (2011) 644

[29] D. J. Walton et al., X-Ray Outflows and Super-Eddington Accretion in the Ultraluminous X-Ray Source Holmberg IX X-1, ApJL 773 (2013) L9

[30] M. J. Middleton et al., Broad absorption features in wind-dominated ultraluminous X-ray sources?, MNRAS 438 (2014) L51

[31] L. M. Heil et al., A systematic study of variability in a sample of ultraluminous X-ray sources, MNRAS 397 (2009) 1061

[32] T. E. Strohmayer \& R. F. Mushotzky, Discovery of X-Ray Quasi-periodic Oscillations from an Ultraluminous X-Ray Source in M82: Evidence against Beaming, ApJ 586 (2003) L61

[33] P. Mucciarelli et al., A variable Quasi-Periodic Oscillation in M82 X-1. Timing and spectral analysis of XMM-Newton and RossiXTE observations, MNRAS 365 (2006) 1123

[34] D. R. Pasham et al., A 400-solar-mass black hole in the galaxy M82, Natur 513 (2014) 74

[35] T. E. Strohmayer et al. 2007, Quasi-periodic Variability in NGC 5408 X-1, ApJ 660 (2007) 580

[36] V. K. Agrawal \& A. Nandi, Discovery of a quasi-periodic oscillation in the ultraluminous X-ray source IC 342 X-1: XMM-Newton results, MNRAS 446 (2015) 3926

[37] H. Feng et al., Discovery of Millihertz X-Ray Oscillations in a Transient Ultraluminous X-Ray Source in M82, ApJL 710 (2010) L137

[38] H. Feng \& P. Kaaret, Origin of the X-Ray Quasi-periodic Oscillations and Identification of a Transient Ultraluminous X-Ray Source in M82, ApJ 668 (2007) 941

[39] M. R. Goad et al., A deep XMM-Newton observation of the ultraluminous X-ray source Holmberg II X-1: the case against a 1000-M。 black hole, MNRAS 365 (2006) 191

[40] P. Casella et al., Weighing the black holes in ultraluminous X-ray sources through timing, MNRAS 387 (2008) 1707 
[41] T. E. Strohmayer, Discovery of a 115 Day Orbital Period in the Ultraluminous X-Ray Source NGC 5408 X-1, ApJL 706 (2009) L210

[42] D. R. Pasham \& T. E. Strohmayer, Evidence for Quasi-periodic X-Ray Dips from an Ultraluminous X-Ray Source: Implications for the Binary Motion, ApJ 764 (2013) id. 93

[43] F. Grise' et al., A long-term X-ray monitoring of the ultraluminous X-ray source NGC 5408 X-1 with Swift reveals the presence of dips but no orbital period, MNRAS 433 (2013) 1023

[44] D. L. Foster et al., Interpretation of the 115 Day Periodic Modulation in the X-ray Flux of NGC 5408 X-1, ApJ 725 (2010) 2480

[45] F. Grise' et al., X-ray Spectral State is not Correlated with Luminosity in Holmberg II X-1, ApJL 724 (2010) L148

[46] A. K. H. Kong et al., Swift Observations of the Ultraluminous X-ray Source Holmberg IXX-1, ApJ $722(2010) 1816$

[47] F. Pintore et al., Spectral variability in Swift and Chandra observations of the ultraluminous source NGC 55 ULXI, MNRAS 448 (2015) 1153

[48] A. M. Stobbart et al., A dipping black hole X-ray binary candidate in NGC 55, MNRAS 351 (2004) 1063

[49] R. Soria et al., The Birth of an Ultraluminous X-Ray Source in M83, ApJ 750 (2012) id. 152

[50] M. Henze et al., XMMU J004243.6+412519 - a new X-ray transient in M 31 seen with XMM Newton, ATel \#3890 (2012)

[51] P. Esposito et al., Swift observations of the ultraluminous X-ray source XMMU J004243.6+412519 in M31, MNRAS 428 (2013) 2480

[52] M. J. Middleton et al., Bright radio emission from an ultraluminous stellar-mass microquasar in $M$ 31, Natur 493 (2013) 187

[53] S. A. Farrell et al., An intermediate-mass black hole of over 500 solar masses in the galaxy ESO243-49, Natur 460 (2009) 73

[54] K. Wiersema et al., A Redshift for the Intermediate-mass Black Hole Candidate HLX-1: Confirmation of its Association with the Galaxy ESO 243-49, ApJL 721 (2010) L102

[55] N. Webb et al. 2012, Radio Detections During Two State Transitions of the Intermediate-Mass Black Hole HLX-1, Sci 337 (2012) 554

[56] M. Servillat et al., X-Ray Variability and Hardness of ESO 243-49 HLX-1: Clear Evidence for Spectral State Transitions, ApJ $\mathbf{7 4 3}$ (2011) id. 6

[57] O. Godet et al., Investigating Slim Disk Solutions for HLX-1 in ESO 243-49, ApJ 752 (2012) id. 34

[58] J.-P. Lasota et al., The Origin of Variability of the Intermediate-mass Black-hole ULX System HLX-1 in ESO 243-49, ApJ 735 (2011) id. 89

[59] R. Soria, Eccentricity of HLX-1, MNRAS 428 (2013) 1944 